\title{
ANÁLISIS DE LA SERIE SECULAR DE PRECIPITACIÓN ANUAL DEL OBSERVATORIO FABRA (BARCELONA) (1914-2020)
}

\author{
Javier Martín-Vide \\ jmartinvide@ub.edu \\ M. ${ }^{a}$ Carmen Moreno-García \\ mcmoreno@ub.edu \\ Grupo de Climatología, \\ Departamento de Geografía, Facultad de Geografía e Historia, \\ Universidad de Barcelona, \\ Montalegre, 6, 08001-Barcelona
}

\begin{abstract}
Resumen: Se ha analizado estadísticamente la serie secular de precipitación anual del observatorio Fabra de Barcelona (1914-2020), así como las de cuatro subperiodos (1914-1930, 1931-1960, 1961-1990, 1991-2020). La serie completa no presenta tendencia estadísticamente significativa, siendo, además, parecidos los valores de los parámetros estadísticos de los tres subperíodos de 30 años. Las distribuciones de probabilidad Log-normal y Log-gamma de 2 parámetros, con sesgo positivo, ofrecen un buen ajuste tanto de la serie completa, como de las de los tres treintenios, existiendo en cada caso otras varias que también lo hacen. La distribución Normal, aun siendo aceptable según algunos de los tests de ajuste, es descartada por su simetría y su dominio ilimitado.
\end{abstract}

Palabra clave: Análisis de tendencias, distribución de probabilidad, distribución Log-normal, distribución Log-gamma, observatorio Fabra, precipitación anual.

\section{Analysis of the secular series of annual precipitation of the Fabra observatory (Barcelona) (1914-2020)}

Abstract: The secular series of annual precipitation from the Fabra observatory in Barcelona (1914-2020) has been statistically analyzed, as well as those from four sub-periods (1914-1930, 1931-1960, 1961-1990, 1991-2020).

Recibido: 15-02-2021. Aceptado: 17-03-2021. 
The complete series does not present a statistically significant trend, and the values of the statistical parameters of the three 30-year sub-periods are similar. The 2-parameter Log-normal and Log-gamma probability distributions, with positive bias, offer a good fit for both the complete series and those of the 30-year series, with several others that also do so in each case. The Normal distribution, even though it is acceptable according to some of the fit tests, is ruled out due to its symmetry and its unlimited domain.

Keywords: Annual precipitation, Fabra observatory, Log-gamma distribution, Log-normal distribution, probability distribution, trend analysis.

\section{Introducción}

Como es sabido, la definición de las características del clima de un lugar o de una región requiere de un conjunto amplio de datos de temperatura, precipitación y de otras variables sobre un período de tiempo prolongado: son las llamadas series climáticas, que han de ser largas, continuas, sistemáticas, compuestas por valores precisos y homogéneas.

La Organización Meteorológica Mundial (OMM) establece genéricamente que es necesario un mínimo de 30 años de registros meteorológicos continuos para obtener promedios, las llamadas normales climatológicas, y otros parámetros estadísticos que definan el clima de un lugar. Hay que indicar al respecto que para un elemento como la precipitación en el mundo mediterráneo es recomendable disponer de más de 30 años, dada su elevada variabilidad, mientras que en el caso de la temperatura o de la presión atmosférica con la mitad de años se obtienen promedios aceptables en cuanto a su significación climática.

Con el objeto de comparar el clima de unos lugares y otros se establecieron incluso unos treintenios determinados, los llamados periodos climatológicos internacionales, sobre los que calcular los promedios y otros valores. Así, han sido períodos internacionales 1901-1930, 1931-1960 y 1961-1990, y el pasado 31 de diciembre de 2020 se completó el 1991-2020. Además de permitir la comparación de las series de muchos lugares sobre un mismo período de tiempo, necesaria, por ejemplo, para construir un atlas climático, los períodos internacionales han servido para establecer las anomalías de un año o un mes determinado respecto a las normales del período internacional, convertido así en período de referencia. Esto hoy, en el contexto del cambio climático, es una tarea importante que permite el seguimiento estadístico de la evolución de la temperatura y de otras variables. Precisamente por el clima en cambio que vivimos, entre otras razones, la OMM ha ido flexibilizando la definición de los períodos de referencia y, así, considera simplemente períodos consecutivos de 30 años comenzando el 1 de enero de un año acabado en 1 y finalizando el 31 de diciembre de un año acabado en 0 , como el 
1981-2010, abandonando el concepto de período internacional (OMM, 2019). Se trata ahora de períodos solapados, aunque se recalca la importancia de considerar el último que cumple la anterior condición, y que coincide con el recientemente completado 19912020 (para estudios de cambio climático a largo plazo, el período 1961-1990, que combinó años relativamente fríos, en su primera mitad, con la señal del calentamiento global, en su segunda, permanece también como referencia).

El observatorio Fabra, perteneciente a la Real Academia de Ciencias y Artes de Barcelona (www.racab.es), distinguido por la OMM, en 2018, como estación centenaria de observación a largo plazo, dispone de series climáticas de 107 años de longitud, desde 1914 hasta la actualidad (figura 1). La calidad de las series climáticas de Fabra es excelente, gracias a la labor de sus observadores meteorológicos, al instrumental de precisión, al hecho de que no ha variado su enclave, ni apreciablemente el entorno inmediato (aunque sí el área metropolitana donde se inserta), y al aval de los académicos de la institución científica a la que pertenece el observatorio (Martín-Vide, 2014). Un análisis estadístico de la temperatura media anual ha permitido establecer que su tendencia en el periodo completo 1914-2020 ha sido de +2,1ํㅡ (Martín-Vide, 2021).

En el presente trabajo se analiza la serie pluviométrica anual del período completo con los siguientes objetivos: 1) determinar los valores de los parámetros estadísticos básicos de la serie completa y de los tres treintenios internacionales que comprende y el período inicial de 17 años, es decir, de cuatro subperiodos; 2) establecer las posibles tendencias de la serie completa; 3) comparar las medias y los parámetros de dispersión de los cuatro subperíodos; y 4) determinar las distribuciones de probabilidad de mejor ajuste para la serie completa y para las de sus tres treintenios.

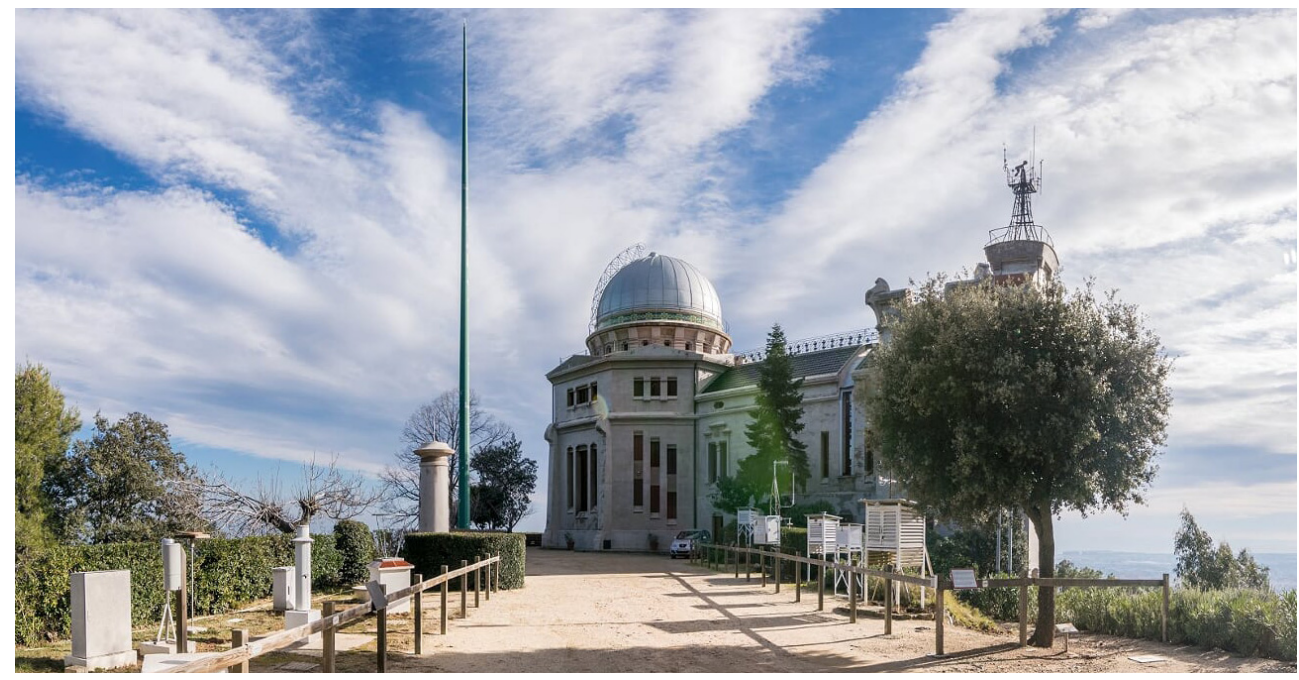

Figura 1. El observatorio Fabra (foto: Alfons Puertas). 


\section{Metodología y lugar de estudio}

Los procedimientos metodológicos empleados han sido, en primer lugar, los propios de la estadística descriptiva, con el cálculo de la media, el rango, los parámetros de dispersión (desviación tipo, varianza y coeficiente de variación), diferentes percentiles y los coeficientes de sesgo y curtosis, tanto para la serie completa como para los 4 subperíodos.

Para el análisis de tendencias se ha empleado el test no paramétrico de Mann-Kendall y el test de Spearman, que no exigen la normalidad de la serie, ni otros requisitos paramétricos. La comparación entre las medias y los parámetros de dispersión de los subperíodos se ha realizado de forma simple, porcentualmente.

Finalmente, la determinación de las distribuciones de mejor ajuste ha conllevado el uso de un gran número de distribuciones de probabilidad, para realizar la elección final, no solo en función de la bondad de los ajustes, sino desde un punto de vista conceptual. Se ha empleado el paquete estadístico EasyFit, de Mathwave, que contiene más de medio centenar de distribuciones de probabilidad. La bondad de los ajustes se establece mediante los tests de Kolmogorov-Smirnov, ${ }^{2}$ (Chi cuadrado) y Anderson-Darling. El paquete estadístico ordena, combinando las tres pruebas, la bondad del ajuste de las diferentes distribuciones de probabilidad. El número de clases $(k)$ impuesto al paquete estadístico ha sido el que provee la regla de Sturges: $k=1+\log _{2} n$, o, lo que es lo mismo, $1+3,322 \log n$, siendo $n$ el número de años de la serie considerada (Sturges, 1926), que, para 107 años, es 8, para 30 años, 6, y para 17 años, 5. Por otra parte, se han mantenido los límites de clases que establece el paquete estadístico.

Las dos distribuciones de probabilidad preferidas para los ajustes han sido: la Lognormal y la Log-gamma de 2 parámetros. La distribución Log-normal (de 2 parámetros), cuyo dominio es $0<\mathrm{x}<+^{\circ}$, presenta las siguientes funciones de densidad de probabilidad y de distribución acumulada:

$$
\begin{gathered}
f(x)=\frac{\exp \left(-\frac{1}{\left.2((\ln x-\mu) / \sigma)^{2}\right)}\right)}{x \sigma \sqrt{2 \pi}} \\
F(x)=\Phi\left(\frac{\ln x-\mu}{\sigma}\right)
\end{gathered}
$$

siendo $\Phi$ la integral de Laplace. 
La distribución Log-gamma de 2 parámetros tiene el mismo dominio y presenta las siguientes funciones de densidad de probabilidad y de distribución acumulada:

$$
\begin{gathered}
f(x)=\frac{\left.(\ln (x))^{\alpha-1}\right)}{x \beta^{\alpha} \Gamma(\alpha)} \exp \left(-\frac{\ln (x)}{\beta}\right) \\
F(x)=\frac{\Gamma \frac{\ln (x)}{\beta}(\alpha)}{\Gamma(\alpha)}
\end{gathered}
$$

siendo $\Gamma$ la función gamma de Euler.

Los datos analizados pertenecen al observatorio Fabra (41,418ㅇN; 2,124ํㅡ; $410 \mathrm{~m})$, sito en la parte más elevada del municipio de Barcelona, en la vertiente marítima de la sierra de Collserola. Posee un clima del tipo Csa, según la clasificación empírica de Köppen-Geiger, es decir, templado con verano seco y caluroso (Kottek et al, 2006); clima de latitudes medias mediterráneo o subtropical húmedo-seco, según la clasificación genética de Strahler y Strahler (1997); y clima mediterráneo de fachada oriental catalán, según la clasificación fisiográfica de los climas de España de Martín Vide y Olcina (2001), con una temperatura media anual de 14 a $17^{\circ} \mathrm{C}$ y una amplitud media anual con la misma horquilla, y régimen pluviométrico con máximo otoñal y mínimo estival.

\section{Resultados y discusión}

\subsection{Análisis estadístico descriptivo (1914-2020)}

Se han hallado los valores de los parámetros estadísticos básicos de la serie pluviométrica anual completa (1914-2020) del observatorio Fabra, que se presentan en la tabla 1.

La precipitación media anual a partir de la serie de 107 años es de 620,1 mm, con totales anuales extremos de $345,8 \mathrm{~mm}$, en 2015, y 1.122,5 mm, en 1971, valor este que supone 3,2 veces el primero. Visto de otra manera, el año más seco solo recogió el $55,8 \%$ del promedio, mientras que el más lluvioso supuso el 181,0\% de él. La serie es platicúrtica y, según el coeficiente de asimetría, con muy ligero sesgo positivo, aunque luego se verá con más claridad la existencia de asimetría positiva, lo que también puede deducirse de una mediana $(585,1 \mathrm{~mm})$ inferior a la media. El coeficiente de variación superior al 20\% (0,246) delata la filiación mediterránea de la pluviometría del observatorio Fabra (Martín-Vide, 2011). En la cuarta parte de los años el total anual apenas alcanza los $500 \mathrm{~mm}(\mathrm{Q} 1=501,7 \mathrm{~mm})$, mientras que en algo más de otra cuarta parte rebasa los $700 \mathrm{~mm}(\mathrm{Q} 3=708,5 \mathrm{~mm})$. El promedio anual del observatorio Fabra es ligeramente superior al que se registra en el llano de Barcelona, entre 300 y 400 metros por debajo (Rodríguez et al., 1999). 
Tabla 1. Valores de los parámetros estadísticos de la serie pluviométrica anual completa del observatorio Fabra (1914-2020)

\begin{tabular}{|l|c|l|c|}
\hline Parámetros & Valor & Percentil & Valor $(\mathrm{mm})$ \\
\hline Tamaño de la muestra & 107 & Mínimo & 345,8 \\
\hline Rango $(\mathrm{mm})$ & 776,7 & $5 \%$ & 439,48 \\
\hline Media $(\mathrm{mm})$ & 620,11 & $10 \%$ & 465,46 \\
\hline Varianza $\left(\mathrm{mm}^{2}\right)$ & 23233,0 & $25 \%(\mathrm{Q} 1)$ & 501,7 \\
\hline Desviación tipo $(\mathrm{mm})$ & 152,42 & $50 \%($ Mediana) & 585,1 \\
\hline Coef. de variación & 0,2458 & $75 \%(\mathrm{Q} 3)$ & 708,5 \\
\hline Error estándar $(\mathrm{mm})$ & 14,735 & $90 \%$ & 843,82 \\
\hline Asimetría & 1,0026 & $95 \%$ & 964,34 \\
\hline Curtosis & 0,70039 & Máximo & 1122,5 \\
\hline
\end{tabular}

\subsection{Análisis de tendencias}

La serie pluviométrica anual del observatorio Fabra no presentaba tendencia significativa al cumplir un siglo (1914-2013) (Martín-Vide, 2014). Tampoco presenta tendencia monotónica estadísticamente significativa en el periodo completo (1914-2020) (figura 2), según confirma el test no paramétrico de Mann-Kendall y la de Spearman. Puede, entonces, afirmarse que hoy en la vertiente marítima de la sierra de Collserola llueve la misma cantidad que un siglo largo atrás. Este resultado es, de momento, frecuente en el análisis de series pluviométricas seculares en España, por lo que no existen tendencias generales en el país (Castro et al, 2005; Peña-Angulo et al, 2020), aunque en algunas regiones se han producido disminuciones pluviométricas significativas (Esteban-Parra et al, 1998). En períodos más cortos, semiseculares, relativamente recientes pueden encontrarse reducciones significativas de la precipitación en ciertos meses y estaciones, y regiones (González-Hidalgo et al, 2010; Del Río et al, 2011), o ciertos descensos en los valores anuales (Homar et al, 2009; Vicente-Serrano y Rodríguez-Camino, 2017), y hasta tendencias de distinto signo en una misma región (Ruiz Sinoga et al, 2011).

\subsection{Comparación entre períodos}

Se han calculado los valores de los diferentes parámetros estadísticos para los 4 subperíodos considerados, 1914-1930, 1931-1960, 1961-1990 y 1991-2020, que se presentan conjuntamente en la tabla 2. 


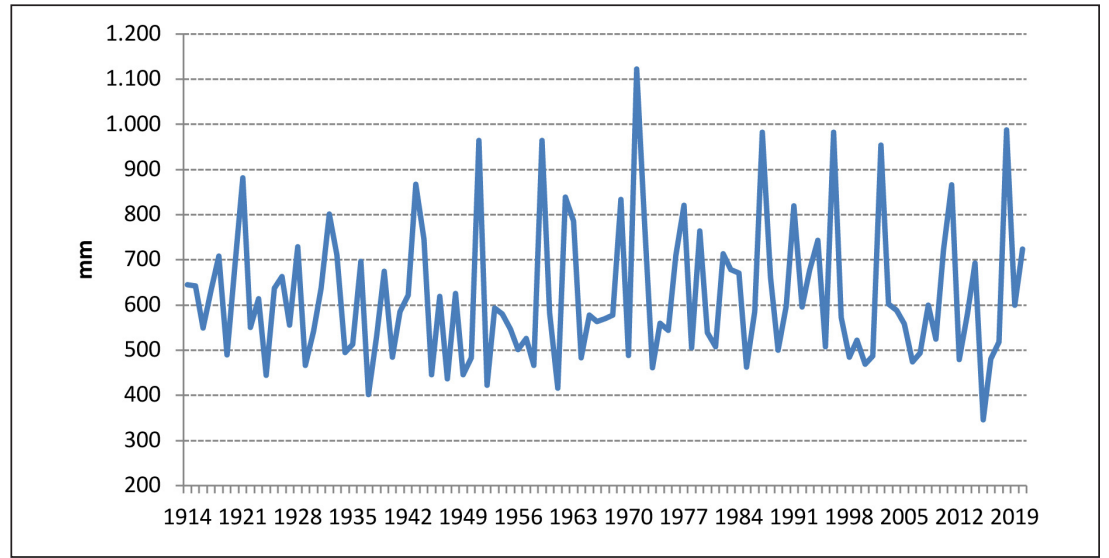

Figura 2. Serie pluviométrica anual completa del observatorio Fabra (1914-2020).

Tabla 2. Valores de los parámetros estadísticos de las series pluviométricas anuales del observatorio Fabra de los subperíodos 1914-1930, 1931-1960, 1961-1990 y 1991-2020

\begin{tabular}{|l|c|c|c|c|}
\hline Parámetros/Percentiles & $1914-1030$ & $1931-1960$ & $1961-1990$ & $1991-2020$ \\
\hline Tamaño de la muestra & 17 & 30 & 30 & 30 \\
\hline Rango & 437,5 & 562,9 & 706,8 & 642,2 \\
\hline Media & 613,46 & 598,87 & 643,7 & 621,53 \\
\hline Varianza & 11637,0 & 22817,0 & 27528,0 & 27085,0 \\
\hline Desviación estándar & 107,87 & 151,05 & 165,91 & 164,58 \\
\hline Coef. de variación & 0,17585 & 0,25223 & 0,25775 & 0,26479 \\
\hline Error estándar & 26,163 & 27,578 & 30,292 & 30,047 \\
\hline Asimetría & 0,62194 & 1,0468 & 1,0717 & 0,9457 \\
\hline Curtosis & 1,0777 & 0,62035 & 1,043 & 0,23673 \\
\hline Min & 443,8 & 401,8 & 415,7 & 345,8 \\
\hline $5 \%$ & 443,8 & 412,8 & 440,51 & 413,62 \\
\hline $10 \%$ & 461,64 & 437,46 & 464,92 & 475,11 \\
\hline $25 \%$ (Q1) & 545,4 & 483,65 & 507,45 & 491,88 \\
\hline $50 \%$ (Mediana) & 634,1 & 581,3 & 581,8 & 584,2 \\
\hline $75 \%$ (Q3) & 671,55 & 679,77 & 769,42 & 721,33 \\
\hline $90 \%$ & 759,22 & 861,11 & 837,87 & 945,33 \\
\hline $95 \%$ & 881,3 & 964,2 & 1045,4 & 984,92 \\
\hline Max & 881,3 & 964,7 & 1122,5 & 988 \\
\hline
\end{tabular}


Los promedios de cada subperíodo no muestran diferencias significativas entre sí. La mayor es de 44,8 mm, entre 1931-1960 y 1961-1990, lo que supone un incremento entre uno y otro subperíodo del 7,5\%. El rango del primer subperíodo es apreciablemente más bajo debido a su brevedad (17 años). Los coeficientes de variación de los tres treintenios superan el 25\%, acorde con la variabilidad pluviométrica en los climas mediterráneos. El del primer subperíodo es anómalamente bajo, explicable, muy probablemente, por la brevedad de su serie. Aunque no pueda atribuírsele significación estadística, los coeficientes de variación se ordenan en el tiempo en orden creciente. Por otra parte, la mediana siempre es inferior a la media, excepto en el primer subperíodo, y el coeficiente de asimetría es positivo en los cuatro subperíodos.

\subsection{Distribuciones de probabilidad de mejor ajuste}

En comparación con los valores de precipitación extremos, como, por ejemplo, las cantidades máximas diarias anuales, para los que se ha estudiado ampliamente las distribuciones de probabilidad de mejor ajuste, las series de totales anuales, así como las estacionales y mensuales, han despertado menor atención (Yue y Hashino, 2007). Uno de los trabajos de referencia es el de Markovic (1965), por el número de series usadas, más de 2.500, de precipitación anual, así como de caudales, del oeste de Estados Unidos y el suroeste de Canadá. Utilizó las distribuciones normal, Log-normal de 2 parámetros, Log-normal de 3 parámetros, Gamma de 2 parámetros y Gamma de 3 parámetros, concluyendo que la Log-normal de 2 parámetros y las gammas ofrecían el mejor ajuste (incluso, la Gamma de 3 parámetros no mejoraba apreciablemente el ajuste dado por la de 2 parámetros). La Gamma de 2 parámetros se ha venido utilizando con bastante generalidad para el ajuste de las distribuciones mensuales de precipitación, en especial en meses poco lluviosos o en climas áridos (Sen y Eljadid, 1999), así como en hidrología (Aksoy, 2000). También la Log-normal se ha seleccionado como la de mejor ajuste de los totales anuales en otros trabajos (Sharma y Sing, 2010). En algunos estudios aparecen otras distribuciones, como la Pearson de tipo III o la Log-Pearson de tipo III en el oeste de Irán (Machekposhti y Sedghi, 2019), esta última, junto con la Johnson SB, la Weibull y otras, en Filipinas, etc. Incluso en un estudio sobre un ámbito tan árido como Sudán sorprende que la distribución Normal, junto con la Gamma, sea la de mejor ajuste, aunque en los lugares hiperáridos deja su puesto a la distribución exponencial (Mohamed e Ibrahim, 2016).

En la figura 3 se presenta el histograma correspondiente a la serie pluviométrica anual completa del observatorio Fabra con los ajustes mediante las distribuciones Lognormal y Log-gamma de 2 parámetros, más la distribución Normal. 


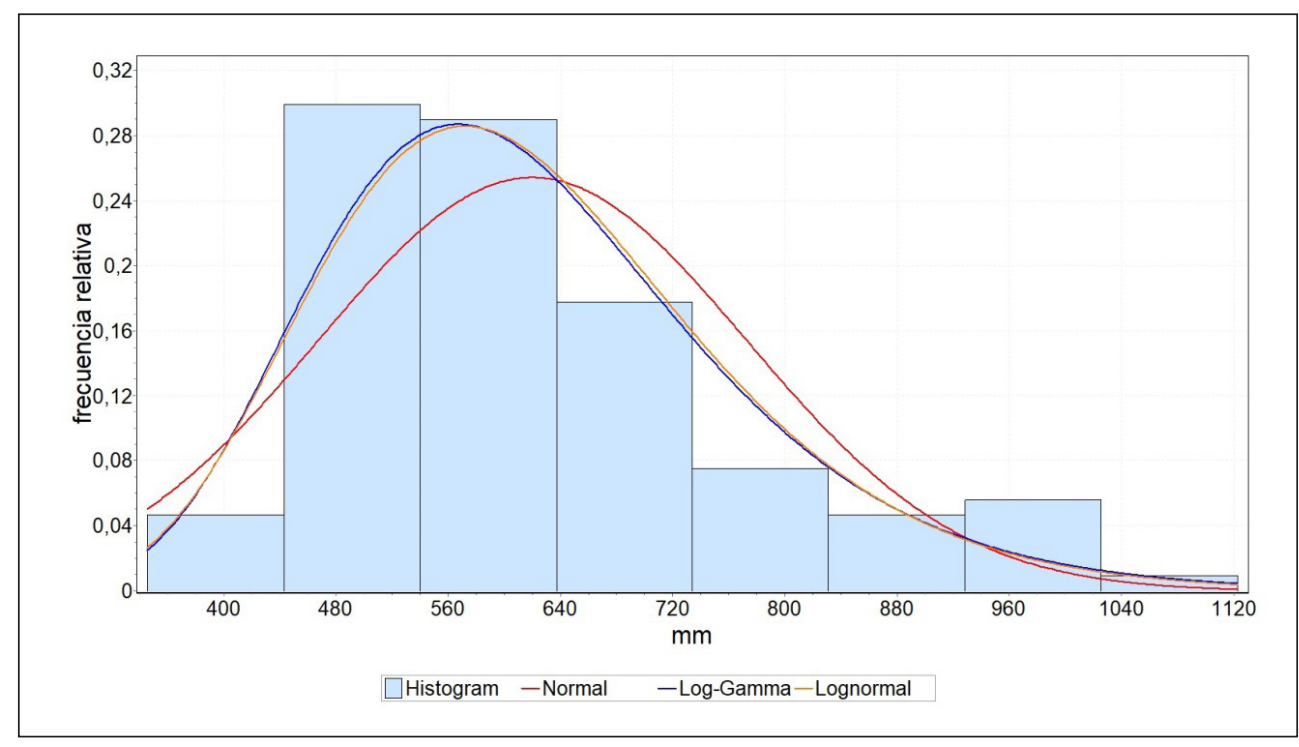

Figura 3. Histograma de frecuencias y ajustes probabilísticos mediante las distribuciones Log-normal, Log-gamma de 2 parámetros y Normal de la serie pluviométrica anual del observatorio Fabra del período 1914-2020.

Los ajustes mediante las distribuciones Log-normal $(\sigma=0,23089 \mu=6,4024)$ y Loggamma de 2 parámetros $(\alpha=761,72 \beta=0,00841)$ son casi idénticos y se adaptan muy bien al sesgo positivo de la distribución empírica. La distribución Normal, aun siendo aceptable según los 3 tests utilizados, debe rechazarse desde un punto de vista conceptual, dado que la distribución empírica claramente no es simétrica. Además, al tratarse de precipitación, los valores de la distribución empírica están acotados inferiormente, siendo, por el contrario, el dominio de la distribución Normal ilimitado (hay que matizar que a nivel anual solo en climas desérticos extremos el límite de $0 \mathrm{~mm}$ puede ser posible algún año).

Existen otras muchas distribuciones que dan un ajuste aceptable para la serie anual completa del observatorio Fabra, incluso mejor, según EasyFit, que las dos elegidas. De hecho, el programa las ordena según un resumen de los tres test, aunque sus correspondientes gráficos muestran en algunos casos separaciones notables del histograma de frecuencias. En otros casos, el ajuste visual que suministran es similar al de las distribuciones Log-normal y Log-gamma de 2 parámetros, como es el caso de la Gamma de 3 parámetros.

En la figura 4 se presenta el histograma correspondiente a la serie pluviométrica anual del treintenio 1931-1960 con los ajustes mediante las distribuciones Log-normal y Log-gamma de 2 parámetros, más la distribución log-Pearson 3. 


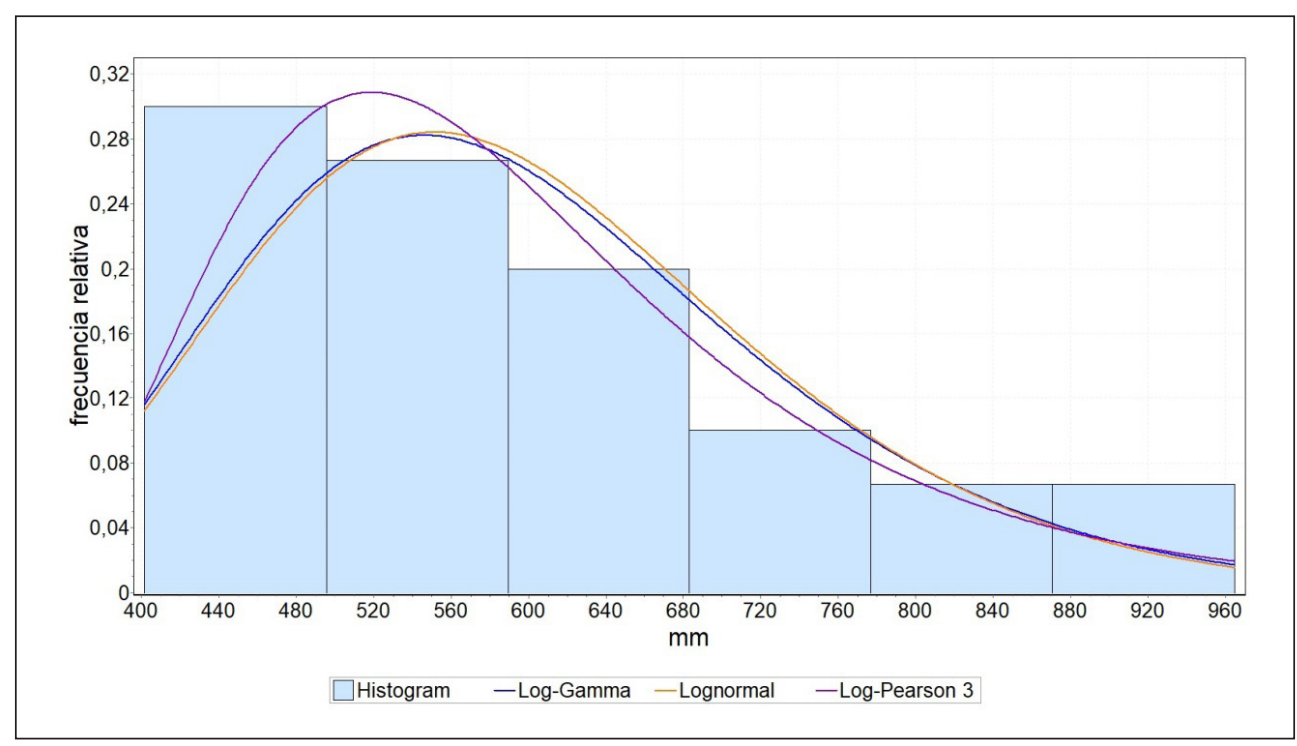

Figura 4. Histograma de frecuencias y ajustes probabilísticos mediante las distribuciones Log-normal, Log-gamma de 2 parámetros y la Log-Pearson 3 de la serie pluviométrica anual del observatorio Fabra del período 1931-1960.

Descartada la ley normal por lo dicho anteriormente, se han elegido entre todas las distribuciones de ajuste la Log-normal $(\sigma=0,23241 \mu=6,367)$ y la Log-gamma de 2 parámetros $(\alpha=725,46 \quad \beta=0,00878)$, y la que EasyFit considera la de mejor ajuste, la Log-Pearson $3(\alpha=12,328 \beta=0,06733 \Upsilon=5,537)$. En efecto, esta última, que, como las otras dos, es una distribución de probabilidad continua y no negativa, parece ofrecer un mejor ajuste visual de la acumulación de valores en las clases inferiores, aunque la elección del número de clases y de los límites de estas pueden variar la bondad de los ajustes.

En la figura 5 se presenta el histograma correspondiente a la serie pluviométrica anual del treintenio 1961-1990 con los ajustes mediante las distribuciones Log-normal y Log-gamma de 2 parámetros, más la distribución Pearson de tipo VI.

Se han elegido de nuevo, entre todas las distribuciones de ajuste, la Log-normal $(\sigma=0,23774 \mu=6,4379)$ y la Log-gamma de 2 parámetros $(\alpha=708,88 \beta=0,00908)$, y la Pearson tipo VI $\left(\alpha_{1}=79,649 \quad \alpha_{2}=23,6 \beta=182,15\right)$, que, entre alguna otra, ofrece un ajuste visual similar a las dos primeras. Esta última distribución de probabilidad también es continua y no negativa.

En la figura 6 se presenta el histograma correspondiente a la serie pluviométrica anual del treintenio 1991-2020 con los ajustes mediante las distribuciones Log-normal y Log-gamma de 2 parámetros, más la distribución de Burr. 


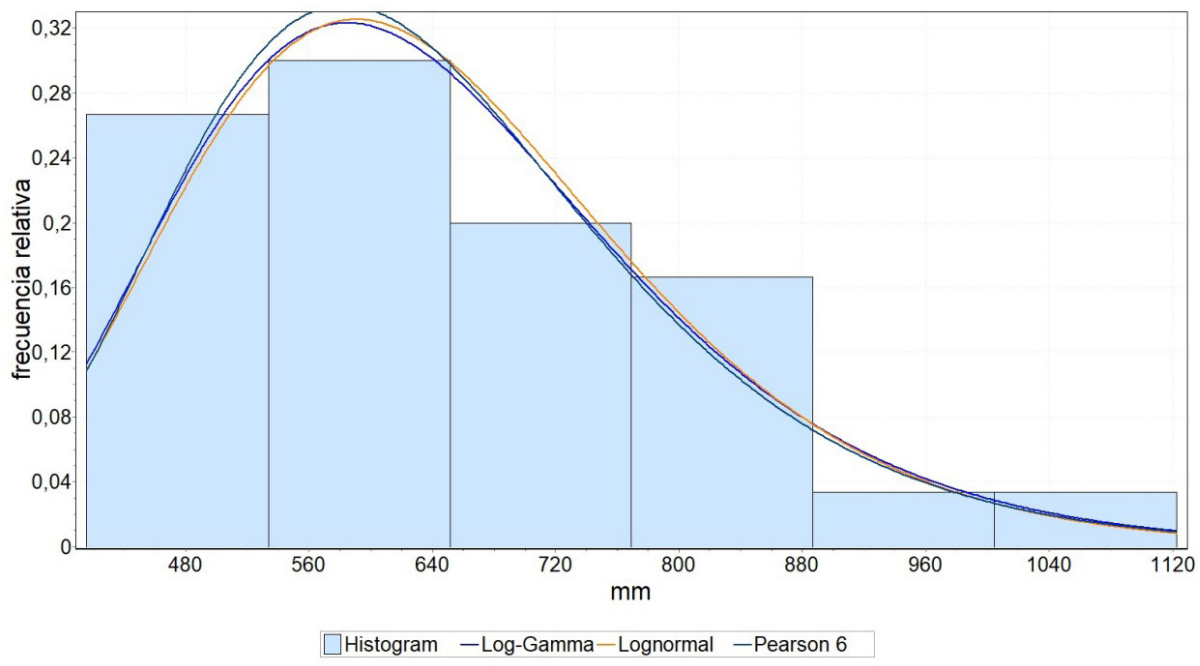

Figura 5. Histograma de frecuencias y ajustes probabilísticos mediante las distribuciones Log-normal, Log-gamma de 2 parámetros y Pearson tipo VI de la serie pluviométrica anual del observatorio Fabra del período 1961-1990.

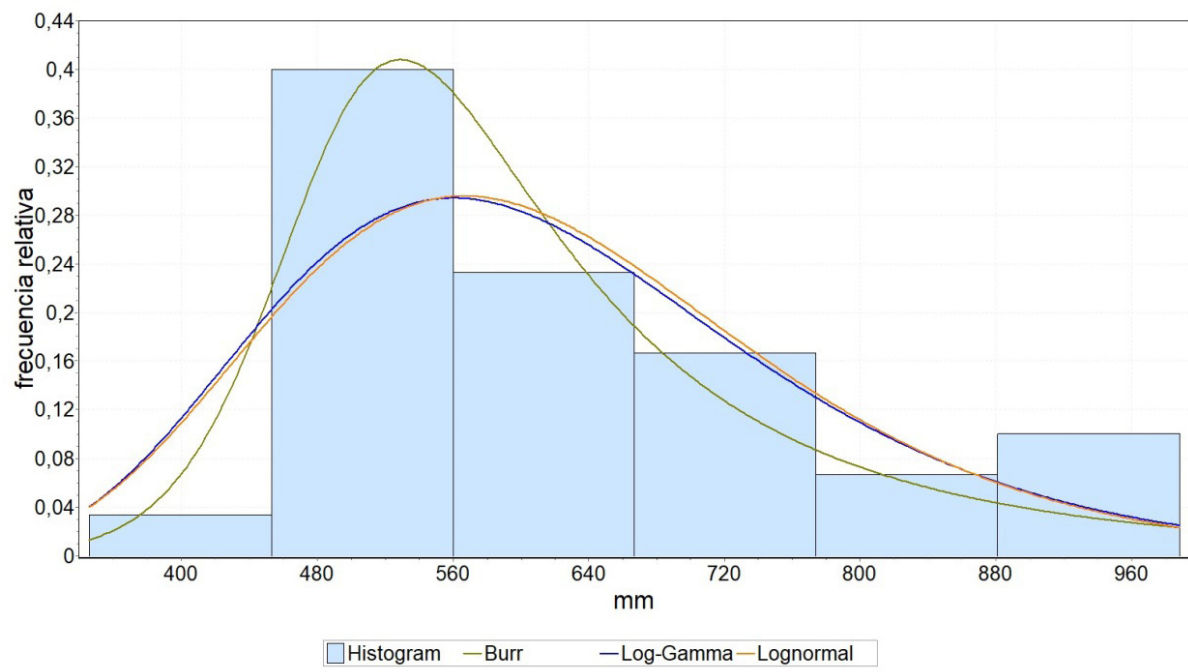

Figura 6 . Histograma de frecuencias y ajustes probabilísticos mediante las distribuciones Log-normal, log-gamma de 2 parámetros y de Burr de la serie pluviométrica anual del observatorio Fabra del período 1991-2020. 
Se han elegido, como en los demás períodos, las distribuciones Log-normal $(\sigma=0,24698$ $\mu=6,4008)$ y Log-gamma de 2 parámetros $(\alpha=649,27 \quad \beta=0,00986)$, y la de Burr $(k=0,34545$ $\alpha=12,698 \beta=497,64)$, que es la de mejor ajuste según EasyFit. Esta última distribución de probabilidad es continua y no negativa, como las otras dos.

En resumen, las distribuciones Log-normal y Log-gamma de 2 parámetros ajustan bien todas las distribuciones de frecuencia analizadas, aunque existen otras varias que, en algunos casos, pueden ofrecer ajustes similares o superiores.

\section{Conclusiones}

La serie pluviométrica anual del observatorio Fabra (Barcelona), de 107 años de longitud, permite, por su continuidad, o ausencia de lagunas, calidad y homogeneidad, un análisis cronológico secular, que no muestra tendencia estadísticamente significativa.

La precipitación media anual para el período completo (1914-2020) es de 620,1 mm y su coeficiente de variación de $24,6 \%$, acorde con una pluviometría relativamente modesta y con una notable variabilidad interanual, características del ámbito climático mediterráneo.

La comparación de los tres treintenios completos de la serie, 1931-1960, 1961-1990 y 1991-2020, no ha detectado diferencias significativas en el comportamiento pluviométrico.

Se han ajustados numerosas distribuciones de probabilidad a la serie completa, así como a las de los tres treintenios, concluyéndose que las distribuciones Log-normal y Log-gamma de 2 parámetros ofrecen un buen ajuste en todos los casos y con muy pocas diferencias entre sí, mientras que la distribución Normal no es aceptable, aun cuando los tests lo permitan, dado que no contempla la asimetría positiva de los histogramas. Por otra parte, existen otras distribuciones de probabilidad que ofrecen, igualmente, buenos ajustes de las series.

\section{Agradecimientos}

Este trabajo se ha realizado en el marco del proyecto CLICES CGL2017-83866-C32-R del Ministerio de Economía, Industria y Competitividad y del Grupo de Climatología 2017 SGR 1362. La Real Academia de Ciencias y Artes de Barcelona ha suministrado la serie pluviométrica objeto de análisis, a través de los observadores meteorológicos Antonio Gázquez y Alfons Puertas del observatorio Fabra. Igualmente, el trabajo forma parte de los intereses del Institut de Recerca de l'Aigua de la Universidad de Barcelona. 


\section{Bibliografía}

Aksoy, H. (2000). Use of Gamma Distribution in Hydrological Analysis. Turkish Journal of Engineering and Environmental Sciences, 24, pp. 419-428.

Castro, M. de; Martín-Vide, J.; Alonso, S. (2005). El clima de España: pasado, presente y escenarios del clima para el siglo XXI. En: Moreno, J.M. (Coord.) Evaluación Preliminar de los Impactos en España por Efecto del Cambio Climático, (pp. 1-64), Madrid: Ministerio de Medio Ambiente.

Del Río, S.; Herrero, L; Fraile, R.; Penas, A. (2011). Spatial distribution of recent rainfall trends in Spain (1961-2006). International Journal of Climatology, 31, pp. 656-667. http://dx.doi.org/ $10.1002 /$ joc. 2111

Esberto, Ma.D.P. (2018). Probability Distribution Fitting of Rainfall Patterns in Philippine Regions for Effective Risk Management. Environment and Ecology Research, 6(3), pp. 178-186. http:// dx.doi.org/10.13189/eer.2018.060305

Esteban-Parra, M.J.; Rodrigo, F.S.; Castro-Diez, Y. (1998). Spatial and temporal patterns of precipitation in Spain for the period 1880-1992. International Journal of Climatology, 18, pp. 1557-1574. http://dx.doi.org/10.1002/(SICI)1097-0088(19981130)18:14<1557::AID-JOC328>3.0.CO;2-J.

González-Hidalgo, J.C.; Brunetti, M.; De Luis, M. (2010). Precipitation trends in Spanish Hydrological Divisions, 1946-2005. Climate Research, 43, pp. 215-228. http://dx.doi.org/10.3354/cr00937

Homar, V.; Ramis, C.; Romero, R.; Alonso, S. (2009). Recent trends in temperature and precipitation over the Balearic Islands (Spain). Climatic Change, 98, 199. https://dx.doi.org/10.1007/s10584009-9664-5

Machekposhti, K.H. y Sedghi, H. (2019). Determination of the Best Fit Probability Distribution for Annual Rainfall in Karkheh River at Iran. International Journal of Environmental and Ecological Engineering, 13(2), pp. 69-75. http://dx.doi.org/10.5281/zenodo.2571967

Markovic, R.D. (1965). Probability of best fit to distributions of annual precipitation and runoff. "Hydro. Paper no. 8", Colorado State Univ., Fort Collins.

Mohamed, T.M. e Ibrahim, A.A.A. (2016). Fitting Probability Distributions of Annual Rainfall in Sudan. SUST Journal of Engineering and Computer Sciences, 17(2), pp. 34-39.

Kottek, M.; Grieser, J.; Beck, C.; Rudolf, B.; Rubel, F. (2006). World map of the Köppen-Geiger climate classification updated. Meteorologische Zeitschrift, 15, pp. 259-263. https://dx.doi.org/ 10.1127/0941-2948/2006/0130

Martín-Vide, J. (2011). Estructura temporal fina y patrones espaciales de la precipitación en la España peninsular. Memorias de la Real Academia de Ciencias y Artes de Barcelona, 1030, LXV, 3, pp. 119-162.

Martín-Vide, J. (2014). Les sèries pluviomètriques centenàries de l'observatori Fabra (1914-2013). En: Martín Vide, J. (Coord.) La Reial Acadèmia de Ciències $i$ Arts de Barcelona i el seu Observatori Fabra, precursors de l'observació meteorològica moderna. Les sèries pluviomètriques centenàries, (pp. 67-86), Barcelona: Reial Acadèmia de Ciències i Arts de Barcelona.

Martín-Vide, J. (2021). Collserola tiene ahora la temperatura del centro de Barcelona de hace 40 años. La Vanguardia. Natural, 12 enero 2021. https://www.lavanguardia.com/natural/cambio-climatico/20210112/6180885/collserola-temperatura-centro-barcelona-40-anos.html 
Martín-Vide, J. y Olcina, J. (2001). Climas y tiempos de España. Madrid: Alianza Editorial.

OMM (2019). WMO Climatological Normals https://www.wmo.int/pages/prog/wcp/wcdmp/ GCDS_1.php\#: :text=The $\% 20$ most $\% 20$ significant $\% 20$ of $\% 20$ these, $1961 \% 2 \mathrm{D} 1990 \% 2 \mathrm{C} \% 20$ and $\% 2$ 0in\%20the

Peña-Angulo, D.; Vicente-Serrano, S.M.; Domínguez-Castro, F.; Murphy, C.; Reig, F.; Tramblay, Y.; Trigo, R.M.; Luna, M.Y.; Turco, M.; Noguera, I.; Aznárez-Balta, M.; García-Herrera, R.; Tomas-Burguera, M.; El Kenawy, A. (2020). Long-term precipitation in Southwestern Europe reveals no clear trend attributable to anthropogenic forcing. Environmental Research Letters, 15, 094070. https://dx.doi.org/10.1088/1748-9326/ab9c4f

Rodríguez, R.; Llasat, M.C.; Wheeler, D. (1999). Analysis of the Barcelona precipitation series 18501991. International Journal of Climatology, 19, pp. 787-801. http://dx.doi.org/10.1002/(SICI) 1097-0088(19990615)19:7<787::AID-JOC382>3.0.CO;2-2

Ruiz Sinoga, J.D.; García Marín, R.; Martínez Murillo, J.F.; Gabarrón Galeote, M.A. (2011). Precipitation dynamics in southern Spain: Trends and cycles. International Journal of Climatology, 31, pp. 2281-2289. http://dx.doi.org/10.1002/joc.2235

Sen, Z. and Eljadid, A.G. (1999). Rainfall Distribution Function for Libya and Rainfall Prediction. Hydrological Sciences Journal, 44(5), pp. 665-680. https://dx.doi.org/10.1080/02626669909492266

Sharma, M.A. y Singh, J.B. (2010). Use of Probability Distribution in Rainfall Analysis. New York Science Journal, 3(9), pp. 40-49.

Strahler, A. N. y Strahler, A. H. (1997). Geografía Física. Barcelona: Omega.

Sturges, H. (1926). The choice of a class-interval. J. Amer. Statist. Assoc., 21, pp. 65-66.

Vicente-Serrano, S.M. y Rodríguez-Camino, E. (2017). Observed atmospheric trends in the Iberian Peninsula, "CLIVAR Exchanges", nำ 73.

Yue, S. y Hashino, M. (2007). Probability distribution of annual, seasonal and monthly precipitation in Japan. Hydrological Sciences Journal, 52(5), pp. 863-877. http://dx.doi.10.1623/hysj.52.5.863 